\title{
Uma comparação entre crianças e adolescentes com enurese noturna primária: impacto e problemas de comportamento
}

\author{
A comparison between children and adolescents \\ with primary nocturnal enuresis: impact \\ and behavior problems
}

Mariana Castro ARANTES

Edwiges Ferreira de Mattos SILVARES

\section{Resumo}

Esse estudo teve como objetivo comparar crianças e adolescentes com enurese noturna primária quanto ao impacto da experiência da enurese e à presença de problemas de comportamento. A pesquisa foi conduzida com um grupo de crianças $(n=76)$ e adolescentes $(n=26)$ inscritos no Projeto Enurese do Instituto de Psicologia da Universidade de São Paulo. Solicitou-se às mães que preenchessem a Lista de Verificação Comportamental para Crianças e Adolescentes. As crianças e os adolescentes responderam a uma entrevista e a partir de suas respostas foram atribuídos graus de impacto da enurese. Tanto nos escores de problemas de comportamento quanto nos graus de impacto não foram encontradas diferenças significativas entre crianças e adolescentes. Os resultados indicaram apenas tendência de as crianças apresentarem uma maior freqüência de escores na faixa clínica e de serem atribuídos graus de impacto maiores às respostas fornecidas pelos adolescentes.

Unitermos: adolescentes; crianças; enurese; problemas de comportamento.

\begin{abstract}
This study aimed to compare children and adolescents with primary nocturnal enuresis in regards of the impact of the experience of enuresis and the presence of behavior problems. The study was conducted on children $(n=76)$ and adolescents $(n=26)$ registered in the Projeto Enurese from the Psychology Institute of the Universidade de São Paulo. Their mothers were asked to fill out the Child Behavior Checklist. The children and adolescents were engaged in a structured interview. The answers were associated to different degrees of enuresis impact. There wasn't significant association between age group and degree of nocturnal enuresis impact, only a tendency to find greater degrees of impact among adolescents. On regards of Child Behavior Checklist scores, there were no significant differences between children and adolescents. The results only pointed to a slight tendency of children to present a greater frequency of clinical scores in all Child Behavior Checklist problem scales.
\end{abstract}

Uniterms:adolescents; children; enuresis; behaviorproblems.

$\boldsymbol{\nabla} \boldsymbol{\nabla} \boldsymbol{\nabla} \boldsymbol{\nabla}$

1 Bolsista FAPESP, Universidade de São Paulo, Pós-Graduação em Psicologia Clínica. São Paulo, SP, Brasil.

2 Universidade de São Paulo, Instituto de Psicologia, Departamento de Psicologia Clínica. Av. Prof. Mello Moraes, 1721, Cidade Universitária, 05508-030, São Paulo, SP, Brasil. Correspondência para/Correspondence to: E.F.M. SILVARES. 
A enurese noturna pode ser um problema significativo para a criança ou adolescente e sua família. Afirma-se com freqüência que as implicações da enurese podem tornar-se mais graves com o aumento da idade, mas são poucos os estudos que procuram investigar objetivamente os efeitos da idade sobre a repercussão da enurese do ponto de vista do enurético.

Em um levantamento bibliográfico, foram encontradas apenas quatro pesquisas com o objetivo de explorar o impacto da enurese desde a perspectiva dos enuréticos (Butler, Redfern \& Forsythe, 1990; Morison, 1998; Ng \& Wong, 2004; van Tijen, Messer \& Namdar, 1998; Wolanczyk, Banasikowska, Zlotkowski, Wisniewski \& Paruszkiewicz, 2002b), e duas pesquisas em que essa investigação não foi realizada como um dos principais objetivos (Redsell, Collier \& Evans, 2003a; Redsell, Collier, Garrud, Evans \& Cawood, 2003b). Em quatro desses estudos, foram encontradas diferenças no impacto da enurese em função do aumento da idade.

Butler et al. (1990) investigaram, por meio de uma entrevista estruturada realizada com a criança, sentimentos e pensamentos referentes à enurese noturna. As crianças mais novas tenderam a perceber seu problema em termos não psicológicos, preocupando-se com as conseqüências imediatas, sensações, cheiro, higiene e contingências que seguem o molhar-se. As crianças mais velhas apontaram com maior freqüência para os efeitos sociais, emocionais e sobre os pais, ou seja, pareceram construir seu problema mais em termos psicológicos.

Van Tijen et al. (1998) desenvolveram um instrumento (Critical Life Events Picture Test) para investigar a percepção da enurese noturna em relação a dez outros eventos de vida críticos. Cartões ilustrados com dois eventos críticos de vida eram apresentados aos sujeitos, que deveriam indicar qual das duas situações era considerada mais grave. Para as crianças com enurese, o problema ocupou a terceira posição, em gradação de gravidade, logo após divórcio e brigas intensas entre os pais; para os adolescentes, a segunda posição, juntamente com brigas intensas entre os pais, depois de divórcio. Os autores não exploraram, mas parece interessante apontar que, em média, os adolescentes consideraram a enurese na mesma posição que brigas entre os pais, evento que ocupou sempre, também para as crianças e adolescentes do grupo-controle, a segunda 156 posição.
Wolanczyk et al. (2002) investigaram as atitudes de crianças enuréticas em relação a sua doença por meio de uma escala desenvolvida para avaliar sistematicamente as atitudes de crianças quanto a condições físicas crônicas em geral (Child Attitude Toward Illness Scale-CATIS). Essa escala compõe-se de 13 perguntas às quais a criança tem de responder de acordo com uma escala Likert de cinco pontos ("Quão justo é que você tenha enurese?" "Quão bem ou mal você se sente em ter enurese?" Com que freqüência você sente que a enurese impede você fazer coisas que você gostaria de fazer?). Escores indicativos de atitudes menos favoráveis foram encontrados nos grupos de enuréticos mais velhos, sugerindo que as atitudes referentes à enurese podem tornar-se mais negativas em função da idade.

Redsell et al. (2003b), com o objetivo de caracterizar a população de crianças em um serviço comunitário de enurese, aplicaram a escala de Impacto da Enurese desenvolvida por Butler (1994). Essa escala consiste de 17 afirmações, tais como "Minha mãe tem roupa demais para lavar", "Tenho que evitar que meus amigos entrem em meu quarto", "Meu pai ou minha mãe fica bravo(a) comigo", "Sinto-me diferente dos meus amigos". A essas afirmações são apresentadas respostas "não", "às vezes" e "sim", por meio das quais são calculados valores de graus de impacto. Os resultados indicaram uma correlação positiva fraca entre a idade e o escore obtido na escala.

Ng e Wong (2004) pesquisaram, por meio de um questionário estruturado, se as crianças apresentavam ou não as seguintes atitudes diante da enurese: evitar falar sobre a enurese com outras pessoas, negar a ocorrência de episódio enurético, evitar dormir fora de casa, usar fralda todas as noites, arrumar a própria cama. Não encontraram diferenças na incidência dessas atitudes em crianças com idades entre sete e dez anos e crianças com mais de dez anos.

O estudo de Morison (1998) distingue-se dos demais estudos por explorar a experiência da enurese desde a perspectiva de crianças e adolescentes por meio de uma abordagem qualitativa. A partir de entrevistas não-estruturadas, foram descritas sete atitudes diante da enurese: falta de preocupação, alegria, pragmatismo otimista, pragmatismo resignado, ambivalência, rejeição ativa e intolerância e falta de esperança resignada. Nesse estudo, não foi feita referência a diferenças de atitude em função da idade. 
Foi encontrada apenas uma pesquisa brasileira que se aproxima da questão do impacto da enurese, mas a perspectiva utilizada foi a de familiares/cuidadores de crianças com enurese, e não a da criança (Soares, Moreira, Monteiro \& Fonseca, 2005).

Os efeitos negativos da enurese em função da idade aparecem também em relação à presença de problemas de comportamento. Uma revisão recente da literatura sobre a presença de problemas de comportamento em crianças enuréticas indicou a existência de dois estudos sugerindo que quanto maior a idade em que o controle do esfíncter é adquirido mais clínicos tornam-se os escores de problemas de comportamento (Baeyens, Roeyers, Vande Walle \& Hoebeke, 2005).

A atitude diante da enurese e a associação entre problemas de comportamento e enurese são aspectos que, além de se mostrarem relacionados ao aumento da idade, aparecem relacionados ao resultado do tratamento para enurese noturna. Algumas atitudes da criança em relação à enurese, tais como falta de preocupação com o problema e presença de problemas de comportamento, aparecem em alguns estudos como fatores preditores de resultados negativos no tratamento para enurese (Butler \& Gasson, 2005).

Tendo em vista a ausência de pesquisas brasileiras sobre o assunto, o presente estudo teve como objetivo comparar crianças e adolescentes com enurese noturna primária quanto ao impacto da experiência da enurese e à presença de problemas de comportamento.

\section{Método}

\section{Participantes}

O estudo foi conduzido em um grupo de clientes ( $n=102)$ com enurese noturna primária inscritos no Projeto Enurese do Instituto de Psicologia da Universidade de São Paulo. Esse grupo consistiu de 76 crianças, na faixa etária entre cinco e dez anos e idade média de 7,95, e 26 adolescentes, na faixa etária entre 11 e 17 anos e média de 13 anos.

\section{Instrumentos e procedimentos}

Solicitou-se às mães que preenchessem a Lista de Verificação Comportamental para Crianças e
Adolescentes (Child Behavior Checklist-CBCL) (Achenbach, 1991). Esse instrumento busca avaliar a competência social e os problemas de comportamento em crianças e adolescentes a partir do preenchimento pelos pais. Os itens de problemas de comportamentos são agrupados em nove escalas: I. Isolamento, II. Queixas Somáticas, III. Ansiedade/Depressão, IV. Problemas Sociais, V. Problemas de Pensamento, VI. Problemas de Atenção, VII. Comportamento Delinqüente, VIII. Comportamento Agressivo e IX. Problemas Sexuais. As escalas I, || e III referem-se aos Distúrbios Internalizantes (DI) e as escalas VII e VIII, aos Distúrbios Externalizantes (DE). A soma dos escores das escalas individuais corresponde ao Total de Problemas de Comportamento (DT).

Os agrupamentos de Distúrbios Internalizantes e Distúrbios Externalizantes refletem associações empíricas entre grupos de escalas que tratam de tipos de problemas de comportamento contrastantes. Os nomes dados às escalas fazem referência ao conteúdo dos itens do CBCL.

Os pontos de corte nos escores determinam as categorias não clínica, limítrofe e clínica para as escalas. Para a soma das escalas de problemas de comportamento (DI, DE e DT), os escores menores do que 60 caem na categoria não clínica; os escores maiores ou iguais a 60 e menores ou iguais a 63, na categoria limítrofe; e os escores maiores que 63, na categoria clínica. Esse instrumento apresenta dados preliminares de validação (Bordin, Mari, \& Caiero, 1995).

As crianças e adolescentes responderam ao roteiro de entrevista elaborado por Butler (Blackwell, 1989). Esse roteiro de entrevista consiste de uma ordem fixa de perguntas abertas. Após as duas primeiras perguntas, solicita-se que a criança faça dois desenhos: um sobre a experiência de acordar com a cama molhada e outro sobre a experiência de acordar com a cama seca. As perguntas agrupam-se em oito áreas: clarificação do problema, como a enurese afeta a vida da criança, descrição da enurese, implicações da enurese, pressão familiar, experiência da enurese, enurese como um segredo, como ficar seco.

Para as crianças, houve o auxílio de um entrevistador treinado, que fazia as perguntas oralmente e registrava as respostas. Os adolescentes e algumas 
crianças mais velhas responderam sozinhos ao roteiro, com a ajuda do entrevistador apenas em casos de dúvida.

Butler et al. (1990) analisaram as respostas dessa entrevista em termos de presença ou ausência de determinadas categorias. Para o presente estudo, foi realizada uma tentativa de relacionar, de modo padronizado, as respostas da entrevista a graus de impacto da enurese. Com base em critérios fixos, três juízes independentes determinaram categorias de impacto, que poderiam variar de zero a quatro, associadas ao conjunto de respostas dadas na entrevista por cada criança ou adolescente. Tomou-se como medida do grau de impacto o valor acordado por, no mínimo, dois desses três juízes. Quando não houve essa concordância mínima de dois entre três juízes, um quarto juiz foi solicitado a avaliar, de modo que, nesses poucos casos, a medida do grau de impacto foi o valor acordado entre dois de quatro juízes.

Esse estudo é derivado de um trabalho maior, de autoria de Costa (2005), que foi submetido à aprovação pelo Comitê de Ética em Pesquisa: A enurese noturna na adolescência e a intervenção comportamental em grupo versus individual com uso de aparelho nacional de alarme. Dissertação de mestrado, Instituto de Psicologia, Universidade de São Paulo.

\section{Resultados}

\section{Impacto da enurese noturna}

Não foi encontrada associação significativa entre faixa etária e grau de impacto da experiência da enurese noturna $\left(\chi^{2}=6,817, p=0,146\right)$, apenas uma tendência de serem atribuídos graus de impacto maiores às respostas fornecidas pelos adolescentes. Para 50\% das crianças e 73,07\% dos adolescentes foram determinados os maiores graus de impacto (valores iguais a ou maiores que três).

\section{Problemas de comportamento}

Em termos de média de escores no $C B C L$, não foram encontradas diferenças significativas entre crianças e adolescentes. A Tabela 1 mostra, para cada um dos índices do CBCL, a porcentagem de escores considerados dentro da categoria clínica. Os resultados indicam uma pequena tendência de as crianças apresentarem escores clínicos em todos os índices do $\mathrm{CBCL}$. Enquanto a porcentagem de adolescentes que apresentaram escores clínicos nas escalas referentes a Distúrbios Externalizantes foi de 30,8\%, a porcentagem de crianças foi de 40,8\%. Essa diferença é um pouco maior quando se trata dos escores relativos a Distúrbios Totais: 38,5\% dos adolescentes e 52,6\% das crianças apresentaram escores dentro da faixa clínica.

Tabela 1. Porcentagens de escores de crianças e de adolescentes dentro da categoria clínica para cada um dos índices do $C B C L$.

\begin{tabular}{lccc}
\hline & DI & DE & DT \\
\hline Crianças $(n=76)$ & $30(39,5 \%)$ & $31(40,8 \%)$ & $40(52,6 \%)$ \\
Adolescentes $(n=26)$ & $9(34,6 \%)$ & $8(30,8 \%)$ & $10(38,5 \%)$ \\
$p^{*}$ & 0,660 & 0,364 & 0,212 \\
\hline
\end{tabular}

DI: Distúrbios internalizantes; DE: Distúrbios externalizantes; DT: Distúrbios totais; CBCL: Lista de verificação comportamental para crianças e adolescentes; *Valor-p do teste Qui-Quadrado.

\section{Discussão}

Os resultados apontaram pouca diferença no grau de impacto da enurese noturna entre crianças e adolescentes, havendo apenas uma pequena tendência de que adolescentes expressassem maior grau de impacto. Nos quatro estudos específicos encontrados que procuraram investigar o impacto da enurese noturna e tomaram a idade como uma variável (Butler et al., 1990; Ng \& Wong, 2004; van Tijen et al., 1998; Wolanczyk et al., 2002), a forma com que o impacto foi avaliado não permite comparações diretas com o presente estudo. Os três primeiros estudos encontraram diferenças em função da idade. Os resultados do estudo de Ng e Wong (2004), indicando não haver diferença das reações à enurese com a idade, e os resultados de Redsell et al. (2003b), apontando apenas para uma pequena correlação positiva dos escores na escala em função da idade, aproximam-se dos dados encontrados nesse estudo.

A tentativa empreendida de transformar o discurso de crianças e adolescentes sobre o impacto da enurese noturna em termos quantitativos precisa ser validada empiricamente, o que faz parte dos planos de pesquisa futuros das autoras. Tal validação seria de grande utilidade, haja vista a carência de estudos com o fim de determinar uma medida objetiva da intensidade 
do impacto envolvido na experiência da enurese e empiricamente validada. O único instrumento validado por diversos estudos foi o utilizado por Wolanczyk et al. (2002), que apresenta, entretanto, a desvantagem de não ter sido desenvolvido especificamente para avaliar o impacto da enurese.

Contrastando com outras evidências (Baeyens et al., 2005), os resultados não apontaram uma maior correlação da enurese noturna com problemas de comportamento em função da faixa etária.

Os resultados do presente estudo apresentam as limitações relativas ao tamanho da amostra. A porcentagem de adolescentes enuréticos na população em geral dificulta bastante a consecução desse tipo de estudo. Outra limitação importante da amostra é o fato de tratar-se de crianças e adolescentes que procuraram espontaneamente pelo atendimento oferecido pelo projeto. Moffat (1989), a partir de uma revisão de estudos que compararam crianças enuréticas que procuram tratamento com crianças enuréticas que não procuram, concluiu que crianças que procuram tratamento para enurese encontram-se sob mais estresse e apresentam mais problemas de comportamento do que crianças que não procuram. Esses dados são importantes para se pensar na maior freqüência, ainda que não estatisticamente significante, de escores clínicos no CBCL entre as crianças. É possível levantar a hipótese de que a procura mais precoce por tratamento seja também motivada por outras dificuldades de comportamento.

Assim, pode-se pensar que o tamanho da amostra e o fato de ser derivada de uma população em busca de tratamento para enurese podem ter tido influência sobre os resultados, camuflando possíveis diferenças entre as crianças e os adolescentes com enurese noturna primária. Essas diferenças, indicadas por outros estudos, sugerem que problemas apresentados pelos enuréticos são mais conseqüência do que causa da enurese.

Essa hipótese é reforçada por evidências de que não há diferenças no tempo para aquisição da continência entre enuréticos com problemas psiquiátricos e enuréticos sem problemas (Moilanen et al., 1998). O efeito do aumento da idade sobre a associação entre enurese e problemas de comportamento não parece, portanto, poder ser explicado pelo fato de que problemas de comportamento constituam-se obstáculos à remissão da enurese.
Outro tipo de evidência que reforça essa hipótese são os resultados de estudos que apontam melhoras nos problemas de comportamento após o tratamento para enurese. Hirasing, van Leerdam, Bolk-Bennink e Koot (2002) estudaram os efeitos do tratamento para enurese sobre os problemas de comportamento das crianças enuréticas. Das crianças que apresentavam escores de Distúrbios Totais no CBCL na faixa clínica e limítrofe, 58\% passaram a apresentar escores na faixa normal após tratamento bem-sucedido.

\section{Considerações Finais}

Ainda que os resultados não tenham indicado maior grau de impacto e maior correlação da enurese noturna com problemas de comportamento em função do aumento da idade, considera-se, com base nos resultados de outros estudos, que isso se deva principalmente a limitações da amostra.

A hipótese de que outros problemas que o enurético possa apresentar sejam mais conseqüência do que causa da enurese sugere que a função do tratamento não é apenas solucionar os episódios enuréticos, mas também prevenir ocorrência de outras dificuldades. A possibilidade de que o tratamento da enurese noturna seja também preventivo constitui um motivo a mais para ser procurado pelos pais e difundido pelos serviços de saúde.

\section{Referências}

Achenbach, T. M. (1991). Integrative guide for the 1991 CBCL/4-18, YSR, and TRF Profiles. Burlington: University of Vermont.

Baeyens, D., Roeyers, H., Vande Walle, J., \& Hoebeke, P. (2005). Behavioural problems and attention-deficit hyperactivity disorder in children with enuresis: a literature review. European Journal of Pediatrics, 164 (11), 665-672.

Blackwell, C. (1989). A guide to enuresis: a guide to a treatment of enuresis for professionals. Bristol: Enuresis Resource and Information Center.

Bordin, I. A. S., Mari, J. J., \& Caiero, M. F. (1995). Validação da versão brasileira do Child Behavior Checklist (CBCL) Inventário de comportamentos da infância e da adolescência: dados preliminares. Revista Brasileira de Psiquiatria, 17 (2), 55-66.

Butler, R. J. (1994). Nocturnal enuresis: The child's experience. Oxford: Butterworth-Heinemann. 
Butler, R.J., \& Gasson, S. (2005). Enuresis alarm treatment. Scandinavian Journal of Urology and Nephrology, 39 (5), 349-357.

Butler, R. J., Redfern, E. J., \& Forsythe, W. I. (1990). The child's construing of nocturnal enuresis: a method of inquiry and prediction of outcome. Journal of Child Psychology and Psychiatry, 31 (3), 447-454.

Costa, N. J. D. (2005). A enurese noturna na adolescência e a intervenção comportamental em grupo $x$ individual com uso de aparelho nacional de alarme. Dissertação de mestrado não-publicada, Instituto de Psicologia, Universidade de São Paulo.

Hirasing, R. A., van Leerdam, F. J. M., Bolk-Bennink, L. F., \& Koot, H. M. (2002). Effect of Dry Bed Training on behavioural problems in enuretic children. Acta Paediatrica, 91 (8), 960-964.

Moffat, M. E. K. (1989). Nocturnal enuresis: psychologic implications of treatment and nontreatment. The Journal of Pediatrics, 114 (4 Pt 2), 697-704.

Moilanen, I., Tirkkonen, T., Järvelin, M. R., Linna, S.L., Almqvist, F., Piha, J., Räsanen, E., \& Tamminen, T. (1998). A follow-up of enuresis from childhood to adolescence. British Journal of Urology, 81 (Suppl. 3), 94-97.

Morison, M. J. (1998). Parents' and young people's attitudes towards bedwetting and their influence on behaviour, including readiness to engage in and persist with treatment. British Journal of Urology, 81 (3), 56-66.
Ng, C. F. N., \& Wong, S. N. (2004). Primary nocturnal enuresis: Patient attitudes and parental perceptions. Hong Kong Journal Paediatrics (New Series), 9 (1), 54-58

Redsell, S. A., Collier, J., \& Evans, J. (2003a). Children presenting at UK community enuresis clinics: comparison with hospital-based samples. Scandinavian Journal of Urology and Nephrology, 37, 239-245.

Redsell, S. A, Collier, J., Garrud, P., Evans, J. H. C., \& Cawood, C. (2003b). Multimedia versus written information for nocturnal enuresis education: a cluster randomized controlled trial. Child: Care, Health and Development, 29 (2), 121-129.

Soares, A. H. R., Moreira, M. C. N., Monteiro, L. M. C, \& Fonseca, E. M. G. O. (2005). A enurese em crianças e seus significados para suas famílias: Abordagem qualitativa sobre uma intervenção profissional em saúde. Revista Brasileira de Saúde Materno Infantil, 5 (3), 301-311.

van Tijen, N. M., Messer, A. P., \& Namdar, Z. (1998). Perceived stress of nocturnal enuresis in childhood. British Journal of Urology, 81 (suppl. 3), 98-99.

Wolanczyk, T., Banasikowska, I., Zlotkowski, P., Wisniewski, A., \& Paruszkiewicz, G. (2002). Attitudes of enuretic children towards their illness. Acta Paediatrica, 91 (7), 844-848.

Recebido em: 30/9/2005

Versão final reapresentada em: 26/7/2006

Aprovado em: 5/9/2006 